\title{
Essai de traitement de la péripneumonie bovine par association "sulfones-sulfamides "
}

\author{
par M. MARCQUE
}

Les observations ont porté sur un lot de 4 bœufszébus présentant des signes cliniques typiques de péripneumonie. Les résultats encourageants obtenus nous ont poussé à continuer l'expérimentation sur 22 bovins péripneumoniques.

Observation I. - Bœuf zébu de 290 kilogs. Le jour de l'intervention, la température est de 4105 , la respiration accélérée, courte, avec bruit de gouttelettes vers la fin de l'inspiration. Les muqueuses sont congestionnées. L'animal grince des dents, ne rumine plus. Frissons et tremblements musculaires.

Le traitement institué est le suivant :

1. Coccicid ou " Digalactoside $P P^{\prime}$ DiaminoDiphényl sulfone " en injection intraveineuse de deux ampoulos de $25 \mathrm{cc}$.

$2^{\circ} 1.162 F$ "per os " à raison de 50 grammes à donner en trois fois durant 24 heures.

\section{Deuxième jour :}

- Température : 3905. Légère reprise de l'appétit. La rumination est revenue.

- A noter la régression des symptômes respiratoires.

- Même posologie que précédemment.

\section{Troisième jour :}

- Température redevenue normale. L'aninal mange et rumine. Les symptômes respiratoires ont disparu.

- Une nouvelle injection de deux ampoules de 25 cc. de coccicid est pratiquée.

\section{Quatrième jour :}

- L'animal peut être considéré comme guéri.

Observations II et III. - Zébus du même lot que celui de l'observation I.

La même posologie est adoptée : résultats positifs surprenants dès le troisième jour.
Observation IV. - L'animal est à la période avancée de péripneumonie - membres anténeurs ecartés - percussion douloureuse avec épanchement liquide. A l'auscultation, râles crépitants et souffle tubaire. Bruit de gouttelettes. Amaigrissement accentué.

Pronostic : sombre.

Traitement : Vu la gravité du cas, le traitement au coccicid est effectué comme précédemment en association avec la soluseptazine (solution à $10 \%$ ) en injections sous-cutanées à raison de $50 \mathrm{cc}$. répartis en trois injections dans la journée.

Le deuxième jour : même posologie'.

Dès le troisieme jour, les symptômes respiratoires sont en très nette régression (c'est vrainent spectaculaire comme amélioration). L'animal mange, recommence à ruminer.

Le traitement est continué jusqu'au cinquième jour : $1.162 \mathrm{~F}$ "per os " est substitué aux injections de soluseptazine le troisieme jour.

L'animal peut être considéré comme guéri le cinquième jour.

Suite à ces guérisons vraiment « spectaculaires $»_{1}$ 22 bovins du parc de préadaptation de Nahouré-Nai, atteints de péripneumonie, et isolés, furent traités par association « sulfones - sulfamides ». A noter 19 guérisons, 3 mortalités (ces animaux avaient été traités à la dernière extrémité).

Conclusion. - Le coccicid ou suifone injectable en association avec la sulfamidothérapie semble un produit excellent dans le traitement de la péripneumonie bovine. Outre sa rapidité d'action par voie intraveineuse, il n'est à relever aucun phénomène de choc.

A noter que les guérisons se situent du troisième au cinquieme jour après l'intervention, suivant la gravité du cas. 